\title{
Cryptococcal Aortitis
}

\author{
ANdrew D. LeavitT, M.D., Carol A. KaufFMAN, M.D. Ann Arbor, Michigan
}

F ungal infections of endothelial surfaces are uncommon. Most of the infections are cases of fungal endocarditis involving prosthetic devices. In a recent review of fungal endocarditis, McLeod and Remington [1] were able to find a total of 319 cases in the literature. Candida albicans, other Candida species, Aspergillus, and Histoplasma capsulatum represented 91 percent of the 319 cases. Eleven other fungal species accounted for the remaining 29 cases. Only three of the 319 total cases were caused by Crypiococcus neoformans. Fungal infection of the vascular tree is even less common than fungal endocarditis. We were able to find only one documented case of cryptococcal infection of the aorta [2]. It was documented postmortem. We report a case of $C$. neoformans aortitis that had been present for at least three years in a man who was not an intravenous drug abuser and had no intravascular or cardiac surgery prior to his original infection.

\section{CASE REPORT}

The patient is a 61-year-old retired construction and building demolition worker who was well until December 1982, when he had the onset of bilateral cold feet with associated burning in his legs. In April 1983, he presented to the Ann Arbor Veterans Administration Medical Center (AAVAMC) with symptoms consistent with lower extremity ischemia. An arteriogram of his lower aorta showed severe atheromatous disease with multiple large ulcerated plaques, a $9-\mathrm{cm}$ aneurysmal dilatation of the aorta below the renal arteries, and multiple changes in both legs consistent with subacute and chronic embolic events. He underwent endarterectomy and placement of a $9-\mathrm{cm}$ inverted "Y" Dacron graft in his distal aorta. Over the next 2.5 years, he did well except for unexplained intermittent nausea and vomiting and an associated 10-pound weight loss.

On October 15, 1985, he presented to the AAVAMC with eight hours of generalized abdominal pain; the serum amylase level was $249 \mathrm{IU} /$ liter. He was admitted for possible pancreatitis. Findings on an abdominal radiograph were unremarkable except for excessive stool. The patient was treated with enemas, his abdominal pain decreased, and his amylase level returned to normal within 48 hours. An ultrasound examination of the abdomen showed stones in the gallbladder. The pancreas could not be visualized. The patient had temperatures to $101^{\circ} \mathrm{F}$ for seven days. Six blood cultures yielded no growth, anti-nuclear antibody and rheumatoid factor were negative, and the Westergren sedimentation rate was $70 \mathrm{~mm} /$ hour. Urinalysis showed hematuria and proteinuria. An echocardiogram was negative for vegetations or valvular

From the Division of Infectious Diseases, Department of Internal Medicine, The University of Michigan Medical School, Ann Arbor Veterans Administration Medical Center Ann Arbor Michigan. Requests for reprints should be addressed to Dr Carol A Kauffman Ann Arbor Veterans Administration addressed to Dr, Carol A. Kauffman, Ann Arbor Veterans Administration submitted January 29, 1988, and accepted in revised form April 15, 1988. abnormalities. An abdominal computed tomographic scan gave normal results, except for gall stones, calcified granulomas in the spleen, and findings consistent with infarction of the lower two-thirds of the spleen. The fevers, elevated sedimentation rate, hematuria, proteinuria, splenic infarction, abdominal pain, normal echocardiographic findings, and negative blood cultures raised the question of polyarteritis nodosa. A visceral angiogram was performed, and the only abnormality was a totally occluded inferior splenic artery. No definitive diagnosis was obtained, and the patient was discharged with decreased abdominal pain and no fever. Three months later, he had an elective cholecystectomy because of persistent unexplained abdominal pain.

On March 1, 1986, he presented with the acute onset of a cold, painful right leg. Examination showed absent pulses distally, and angiographic findings were consistent with an acute occlusion of the right common femoral artery. He underwent successful Fogarty embolectomy. Pathologic examination of the embolus showed marked inflammation including occasional multinucleated giant cells. Periodic acid-Schiff stain demonstrated yeast-like organisms, and Meyer's mucicarmine stain showed these organisms to be $C$. neoformans (Figure 1). Four sets of blood cultures yielded no growth, and an echocardiogram showed no valvular abnormalities or vegetations. Three latex agglutination tests of the serum for cryptococcal antigen were negative. Evaluation of the cerebrospinal fluid was negative for cryptococcal antigen, and India ink studies were negative. Protein, glucose, and cell counts performed on the cerebrospinal fluid were normal. The chest radiograph showed no evidence of infection, but did show a large calcified left hilar lymph node.

The pathologic specimen block made three years previously, at the time of the abdominal aortic aneurysm repair, was re-cut. There was marked atheromatous change with mild chronic inflammation. New sections were stained with Meyer's mucicarmine stain and demonstrated budding organisms typical of $C$. neoformans (Figure 2).

To explain the splenic embolus and the lower aortic involvement, we suspected that the proximal aorta served as a primary source within the vascular tree. Angiography of the patient's thoracic aorta demonstrated a $2-\mathrm{cm}$ saccular aneurysm immediately distal to the left subclavian artery. The patient underwent a thoracotomy to remove the aneurysmal tissue and a calcified thoracic lymph node immediately adjacent to the aneurysm. For technical reasons, the aneurysmal dilatation was oversewn but could not be removed. On pathologic evaluation, the lymph node revealed no fungi.

The patient received $1 \mathrm{~g}$ of amphotericin $\mathrm{B}$ with concomitant flucytosine for six weeks. During that time, his Westergren sedimentation rate declined from $78 \mathrm{~mm} /$ hour to $27 \mathrm{~mm} /$ hour. Over the subsequent 20 months, he has done well, with resolution of 


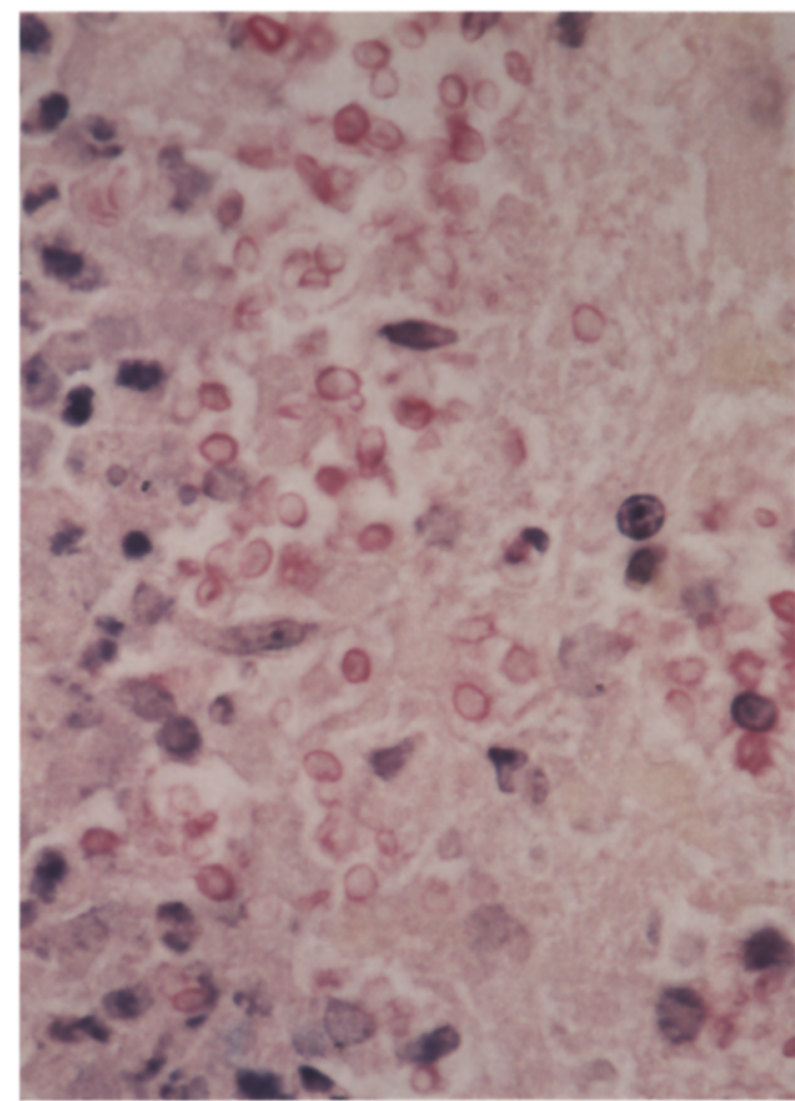

Figure 1. Meyer's mucicarmine stain of femoral embolus demonstrating budding yeast typical of $C$. neoformans (original magnification $X$ 400 , reduced by 55 percent).

his abdominal pain, improvement in his endurance, and stability of his weight. His Westergren sedimentation rate has remained normal.

\section{COMMENTS}

C. neoformans is an encapsulated yeast found in the soil. In humans, it may be a harmless colonizer, but it also may cause serious infection. Although the lung is the portal of entry, disseminated disease is common, with infection evident in the central nervous system, bone, liver, and spleen [3]. Some patients have disseminated cryptococcemia without specific organ involvement but with positive blood culture results [4]. The presumed site of entry of the organism in our patient was the lungs, with infection occurring perhaps during the course of his work as a construction and demolition worker. As is often the case, he had only a calcified lymph node as residual evidence of his pulmonary disease.

Vascular endothelial infections with C. neoformans are quite rare. There have been three case reports of endocarditis and one report of aortitis due to $C$. neoformans in the literature $[2,5-7]$. All have been documented either in very ill patients or at necropsy. The previously reported case of $C$. neoformans aortitis was in a 33-year-old man who presented with a 10 -week history of low back pain [2]. Examination revealed a pulsatile mass below the umbilicus. Three days later,

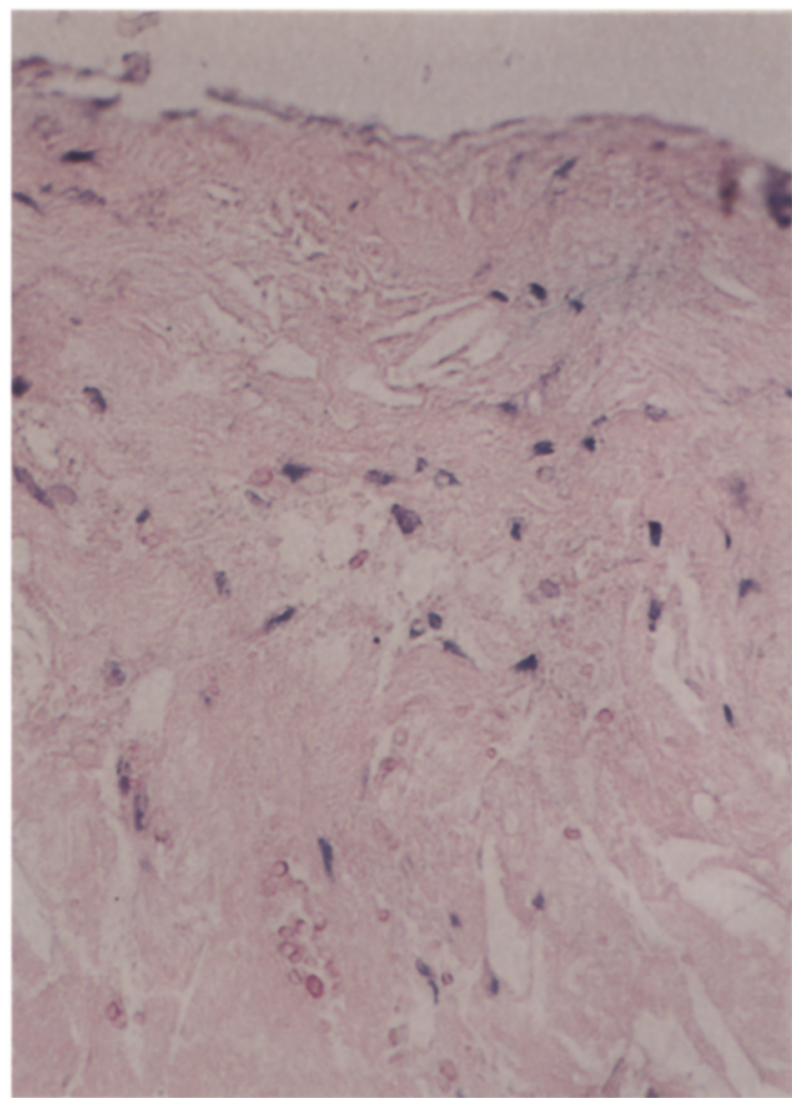

Figure 2. Meyer's mucicarmine stain of a section of the abdominal aortic aneurysm removed three years previously showing budding yeast consistent with $C$. neoformans (original magnification $\times 250$. reduced by 55 percent).

severe abdominal pain and a numb, cold left leg developed. He died two days later. At autopsy, there was a $10 \times 7-\mathrm{cm}$ abdominal aortic aneurysm and perianeurysmal chronic inflammation. The adrenal glands were enlarged, and pathologic examination showed caseous necrosis. Acid-fast stains and cultures were negative, but Cryptococcus was identified histopathologically in the adrenal glands, aortic wall, and epididymis.

Most cases of fungal endocarditis are associated with intravenous drug abuse, previous cardiovascular surgery, or long-term antibiotic therapy. Our patient had none of these risk factors. Cases of fungal aortitis are often associated with atherosclerotic disease, and we believe this played a role in the pathogenesis of disease in our patient. The small thoracic aneurysm in our patient may also have played an important role as the source for an embolus to his spleen. The aneurysm may have been a remnant of his ductus arteriosus, or it may have been associated with the inflammatory lymph node immediately adjacent to the aneurysm.

To the best of our knowledge, this is the first report of cryptococcal aortitis diagnosed premortem and only the second case report of cryptococcal aortitis [2]. Our patient clearly had the cryptococcal organism in contact with his blood stream-found in the aortic wall and in a lower-extremity embolus-yet we were unable to grow the organism from his blood. Other authors have noted difficulty in growing Aspergillus, 
Coccidioides, and Blastomyces from the blood of patients with proven disseminated disease [4]. We did not expect that repeated assays for cryptococcal antigen in serum would be negative. Perhaps an explanation for the negative blood cultures and the negative cryptococcal antigen tests is that this infection was a localized tissue infection (aorta and associated atherosclerotic material) and not truly a disseminated infection. The organisms were clearly identified in the tissue sections. Although the cultures of blood showed no growth, the mucicarmine stain is quite specific for $C$. neoformans, thus establishing the diagnosis of cryptococcal aortitis.

Fungal endocarditis and aortitis are both uncommon disorders, and guidelines for therapy are lacking. With endocarditis, surgical removal of the infected valve is usually necessary. Our patient had aortitis and an infected embolus. We believed we could not remove all areas of his aorta and spleen that, by clinical criteria, were suspected of being infected. We chose to treat with a course of amphotericin B and flucytosine for a total of $1 \mathrm{~g}$ of amphotericin B and six weeks of flucytosine. The patient had a good clinical response to antifungal therapy as noted by normalization of his sedimentation rate, resolution of his weight loss and abdominal pain, and return of his energy level to normal.

\section{REFERENCES}

1. McLeod R, Remington JS: Fungal endocarditis. In: Rahimtoola SH, ed. Infective endocarditis. New York: Grune and Stratton, 1978; $211-290$.

2. Rigdon RH, Kirksey OT: Mycotic aneurysm (cryptococcosis) of the abdominal aorta. Am J Surg 1952; 84: 486-491.

3. Diamond RD: Cryptococcus neoformans. In: Mandell GL, Douglas RG, Bennett $\mathrm{JE}$, eds. Principles and practice of infectious diseases, 2nd ed. New York: John Wiley and Sons, 1985; 1460-1468.

4. Perfect JR, Durack DT, Gallis HA: Cryptococcemia. Medicine (Baltimore) 1983; 62: $98-109$.

5. Jones I, Nassau E, Smith P: Cryptococcosis of the heart. Br Heart J 1965; 27 : $462-464$.

6. Colmers RA, Irniger $W$, Steinberg $\mathrm{DH}$ : Cryptococcus neoformans endocarditis cured by amphotericin B. JAMA 1966; 199: 762-764.

7. Lombardo TA, Rabson AS, Dodge HT: Mycotic endocarditis: report of a case due to Cryptococcus neoformans. Am J Med 1957; 22: 654-670. 